\title{
Modelo de evaluación curricular aplicado por la gestión educativa en relación con el programa del ciclo transición en los jardines de niños y niñas independientes del circuito 05 de la Dirección Regional de Educación de Cartago
}

\section{Curriculum evaluation model applied by educational management in relation to the transition cycle program in the independent kindergartens of the circuit 05, of the Regional Directorate of Education of Cartago}

Recibido 30 abril 2013 • Aceptado 17 mayo 2013 • Corregido 20 mayo 2013

\author{
Rocío Carmona Mendoza? \\ Universidad Estatal a Distancia, Costa Rica \\ rocio.carmona@hotmail.es
}

\begin{abstract}
Resumen. El presente artículo consiste en un breve análisis del modelo de evaluación curricular aplicado por la gestión educativa en relación con el programa educativo del ciclo transición en los jardines de niños y niñas independientes del circuito 05 de la Dirección Regional de Educación de Cartago. El estudio es de tipo descriptivo y fue abordado por medio de un cuestionario. Para este se utilizó el modelo de evaluación CIPP (Contexto Entrada, Proceso y Producto), modelo de evaluación curricular basado en la evaluación cualitativa y el modelo de evaluación de la gestión escolar. Los resultados determinan que el modelo de evaluación CIPP es el que más se asemeja al modelo empleado por la gestión del centro en las tres instituciones en estudio; pero debe ser fortalecido en algunas de sus características y ámbitos, también se evidencia en dos de ellas la presencia de otros modelos, pero deben ser fortalecidos de igual manera.
\end{abstract}

Palabras clave. Administración de la educación, evaluación, evaluación de programas, modelos de programas de evaluación.

Máster en Ciencias de la Educación con énfasis en Administración Educativa de la Universidad de Costa Rica (UCR). Bachiller y Licenciada en Educación Preescolar de la Universidad de Costa Rica (UCR). Actualmente labora para la Universidad Estatal a Distancia (UNED), Costa Rica, como tutora de trabajos finales de graduación para el Departamento de Preescolar. Ha laborado tanto en centros educativos privados como públicos en el nivel de Educación Preescolar. 
Abstract. This article is a brief analysis of curriculum evaluation model applied by the management of education in relation to the transition cycle educational program in independent kindergartens of the Circuit 05 of the Regional Directorate of Education of Cartago. The study is descriptive and was addressed through a questionnaire. For this study, we used the CIPP evaluation model (Context Input, Process and Product), curriculum evaluation model based on qualitative assessment and evaluation model of school management. The results determine the CIPP evaluation model is the most similar to the model used by the management of the center in the three institutions under study, but it should be strengthened in some of its features and areas, also is evident in two of them the presence of other models, which also should be strengthened.

Keywords. Educational Administration, evaluation, program evaluation, evaluation program models.

\section{Introducción}

La intención de este artículo es mostrar al lector un estudio realizado en el 2012, por lo que se procede a explicar algunos conceptos claves que ayudan para entender el proceso de la investigación. Se presenta la relación que tiene la administración de la educación, específicamente la gestión educativa, en la evaluación de programas; en este caso, en el Programa del Ciclo Transición del Ministerio de Educación Pública (MEP, 1996), en tres jardines de niños y niñas independientes del circuito 05 de la Dirección Regional de Cartago. Para ello, se utilizan tres posibles modelos de evaluación de programas que serán descritos en el proceso del artículo.

Este artículo corresponde a una investigación realizada al personal docente y administrativo de las instituciones en estudio, acerca de cómo la gestión educativa del centro ejecuta la evaluación del Programa del Ciclo de Transición, y el modelo que se emplea en cada institución para su evaluación. Al respecto, se encontró que a pesar de que los docentes y la persona administradora no conocían el nombre del modelo, las prácticas realizadas se asemejaban, mayormente, al modelo de evaluación CIPP (Contexto, Entrada, Proceso y Producto) en una de las instituciones, mientras que en las otras dos, con menor grado, los otros dos modelos también estaban presentes de igual manera; para que fueran efectivos necesitaban ser fortalecidos.

El objetivo de la investigación consistía en analizar los diferentes modelos de evaluación curricular aplicada por la gestión educativa de los jardines independientes de la Dirección Regional de Cartago. Para el logro de esta, se preparó un cuestionario que pretendía responder a los tres objetivos propuestos, los cuales son: Definir los modelos de la evaluación curricular aplicados por la gestión educativa a los programas educativos; Identificar los ámbitos de evaluación de programas presentes en el modelo de evaluación curricular y; Describir la percepción que tienen los docentes sobre el modelo de evaluación curricular que aplica la gestión educativa de la institución de los Jardines Independientes del Circuito 05 de la Dirección Regional de Educación de Cartago.

La selección de los jardines independientes se realizó por dos motivos importantes del 
estudio, la primera debía verificarse si el modelo de evaluación de programas del Ciclo de Transición era evaluado por los gestores educativos, y el segundo es que los jardines independientes, poseen su propio administrador, lo cual promueve que el gestor esté directamente trabajando con estos niveles educativos. Cabe destacar que en Costa Rica son pocos los jardines de niños y niñas independientes, por lo que el circuito 05 fue elegido porque tiene tres de ellos, que corresponden al Jardín de Niños República Francesa, Jardín de Niños Carlos Peralta y Jardín de Niños Juan Vázquez de Coronado.

Para el estudio de la evaluación del programa por parte de la gestión, se eligieron tres modelos curriculares entre los cuales están: modelo de evaluación CIPP (Contexto Entrada, Proceso y Producto), propuesto por Stfufellbeam y Shinkfieldm citados por Bausela (2003), modelo de evaluación curricular basado en la evaluación cualitativa del autor Flórez (2003) y el modelo de evaluación de la gestión escolar de Antúnez, Carnicero y Silva (2009).

\section{Administración de la educación}

La administración de la educación toma fuerza en las últimas décadas, es por ella que las instituciones se fortalecen, atendiendo situaciones que le atañen a la educación de forma más integral. A su vez, la administración de la educación observa e investiga acerca de lo pedagógico y administrativo, tratando de resolver los conflictos a través de la toma de decisiones.

El concepto de administración de la educación está íntimamente relacionado con el concepto de educación y el de administración, al juntar ambos conceptos Arroyo (2009) lo define como: "planear, dirigir, ejecutar, controlar y evaluar, los procesos que se desarrollan en las organizaciones educativas, dirigidos a desarrollar las capacidades y el desarrollo de los niños y jóvenes" (p.6).

Otro concepto sobre la administración de la educación es el de Pastrana (1994) citada por Aragón (2005), quien menciona que "administrar significa construir formas de cooperación ordenada y dirigida hacia un fin institucional" (p.5). Estas formas de cooperación se realizan entre el personal docente, los recursos, criterios pedagógicos y las estrategias para llevarlas a cabo.

En efecto, dentro de la administración el profesional en gestión debe velar porque las personas trabajen en conjunto hacia un mismo fin, para esto debe cumplir con ciertas funciones. Aragón (2005), basado en el esquema de Koontz, menciona los siguientes:

- La planeación, que implica la selección de objetivos de la institución educativa y las estrategias y acciones necesarias para alcanzarlos.

- La organización, que conlleva el establecimiento de una estructura intencional de los roles y/o tareas a desempeñar por cada uno de los miembros del personal, buscando el logro 
de metas educativas comunes.

- La dirección que se relaciona con aspectos interpersonales y necesita de las aptitudes necesarias en el director, en cuanto a estilo de liderazgo, motivación y comunicación, para propiciar que cada elemento del personal realice su tarea requerida para contribuir al logro de metas grupales.

- El control, que implica la medición corrección de las actividades de los subordinados para verificar que su desempeño esté de acuerdo con las metas y planes previamente establecidos.

\section{La evaluación}

La evaluación de un centro se puede hacer desde diferentes dimensiones según los procesos que se requiera evaluar como lo son los proyectos institucionales, el personal docente, los programas curriculares, entre otros, con el fin de determinar si las acciones tomadas llevarán al cumplimiento de metas y objetivos propuestos. El concepto de evaluación se refiere, según Escudero y otros (1998) citado por Nieto (2003), a la siguiente:

La evaluación, como una actividad de reflexión, comprensión, valoración y toma de decisiones, es concebida como un proceso transversal a todas y cada una de las actuaciones que ocurren en la educación, así como el conjunto de factores, procesos y agentes que participan en la misma. (p. 265)

Según se ha citado, la evaluación pretende reflexionar sobre todo lo que se hace en un determinado proceso, con el fin de valorar si las actuaciones que se realizan son las más acertadas, si no es así, se realiza la toma de decisiones sobre la marcha del mismo proceso, y no como se pensaba antes, cuando se tenían los resultados finales. Pérez (1995) citado por Pérez (2000), menciona que la evaluación es:

La valoración, a partir de criterios y referencias pre especificados, de la información técnicamente diseñada y sistemáticamente recogida y organizada, sobre cuántos factores relevantes integran los procesos educativos para facilitar la toma de decisiones de mejora. (p. 269)

En relación con la cita del autor anterior, incluye el término que corresponde a que esa valoración se hace sobre criterios y referencias específicas, es decir, debe existir un plan, objetivos, metas, estrategias diseñadas con anterioridad, que permitan al evaluador reconocer si se está llevando a cabo el rumbo que se desea en cuanto a lo que se quiere lograr.

Cabe señalar que la evaluación de la institución permite juzgar la calidad de la educación que se está llevando a cabo en ese lugar, en relación con esto, Ruíz (1998) menciona que la evaluación "tiene la misión de ayudar a mejorar la calidad y la eficacia de la acción educativa, 
proporcionando la información necesaria para la toma de decisiones" (p.45).

Según se observa, la evaluación es la encargada de valorar si los criterios seleccionados están respondiendo a las necesidades de la educación conforme el contexto donde se encuentra la institución, de aquí, la importancia que existe de evaluar constantemente lo que se hace en el centro educativo.

Con respecto de lo anteriormente descrito, el estudio pretende evaluar lo que se refiere al programa, en este caso, si existe en los centros educativos una evaluación de programas que permita la mejora de la educación en la institución observada.

El orden en que se analiza la información del estudio en lo que a las categorías de análisis se refiere, se realizó de acuerdo con el objetivo general: "Analizar los diferentes modelos de evaluación curricular aplicada por la Gestión Educativa de los Jardines Independientes del Circuito 05 de la Dirección Regional de Educación de Cartago". Para esto el estudio abarcó tres objetivos específicos planteados: el primero se refiere a las características del modelo de evaluación de programas que las docentes y directora consideran están presentes en el centro educativo donde laboran. El segundo hace referencia al ámbito de los modelos de evaluación que se identifican como mayormente aplicados por la gestión del centro y, por último, se determina la percepción que se tiene en cuanto al modelo aplicado por la gestión educativa.

La percepción se entiende, dentro de esta investigación, como la valoración que tienen las docentes del centro educativo en cuanto a:

- Diseño del plan que responde a las necesidades del niño desde el programa de preescolar.

- Proceso de evaluación del plan para mejorar los aprendizajes.

- Participación docente en la toma de decisiones que favorezcan la mejor aplicación del programa preescolar.

- Participación de la familia y comunidad.

- Resultados de logros.

- Limitaciones.

- Metas y objetivos.

Para el análisis de los modelos se considera que la evaluación de programas es un "dispositivo de principios que orientan la intención evaluadora y las indicaciones en relación con los ámbitos de evaluación, con los momentos, las fuentes e instrumentos que se pueden utilizar, los procedimientos y los agentes que intervienen en ellos" (Antúnez et al., 2009, p.125). 
En relación con lo anterior, la evaluación de programas observa características como diagnóstico, plan institucional, metas y objetivos, contenidos, seguimiento del plan por medio de un proceso, aprovechamiento de recursos tanto humanos como materiales, entre otros. Cada modelo se inclina por algunos aspectos más que otros y es por ello que se destacan como diferentes a la hora de ejecutarse en la institución.

\section{Evaluación de programas.}

La evaluación de programas según Pérez (1995) citado por Pérez (2000), se puede definir como:

Un proceso sistemático, diseñado intencional y técnicamente, de recogida de información rigurosa - valiosa, válida y fiable - orientado a valorar la calidad y los logros de un programa, como base para la posterior toma de decisiones de mejora, tanto del programa como del personal implicado y, de modo indirecto, del cuerpo social en que se encuentra inmerso. (p.12)

Según lo anterior, la evaluación de programas busca mejorar la calidad de la educación, a través de la recogida de datos, para la toma de decisiones en cuanto al personal, el mismo programa, y los demás factores que integran el centro escolar. Asimismo, Romero (2001) menciona "la evaluación de los centros educativos de los programas o de los proyectos debe orientarse a determinar la valoración para la toma de decisiones" (p. 54).

En efecto, como parte de la administración de centros educativos, la evaluación de todo lo que se hace y en miras a mejorar la calidad de la educación, es de suma importancia en todos los ámbitos anteriores. En la relación que hay en cuanto a evaluación y calidad, Correa (2004) lo explica de la siguiente manera "es tan estrecha la relación calidad - evaluación que el uno no se puede dar sin el otro. Si se diera el caso de no incluir uno de ellos, el otro se da de manera implícita en el utilizado" (p. 91).

En concordancia, la evaluación por sí misma permite que la educación mejore hacia una educación de calidad y evaluación en un sentido tradicional y sencillo, según Correa (2004) "es aquella que lo considera como conjunto de actividades, mediante programas que delimitan el diseño, los recursos y el tiempo. La exigencia del modelo se centra en contrastar el proyecto original con los logros alcanzados" (p. 91).

Por su parte, Martín (1994) citado por Romero (2001), señala que "la evaluación intenta contribuir a mejorar un programa tanto directa como indirectamente: por medio de una interacción directa con los participantes en el programa y posibilitando la interacción entre los participantes en el programa y sus audiencias" (p. 54). 
En relación con lo anterior, se observa que la evaluación siempre será en mejora de la calidad y es el profesional en gestión de la educación quien elige el modelo que desea aplicar para evaluar, ya sea el ámbito administrativo, sociopolítico, académico o cultural de su institución, por medio de la interacción activa de los participantes dentro de los programas.

\section{Modelos de evaluación de programas.}

Dentro del estudio se tomaron en cuenta tres diferentes modelos de evaluación que permiten promover la evaluación de los programas de manera coherente, entre estos están el modelo CIPP, modelo curricular y el modelo de gestión escolar.

El modelo CIPP permite evaluar el contexto, para tomar decisiones sobre la designación de metas; la evaluación de entrada como ayuda para dar forma a las propuestas; la evaluación de proceso, como una guía para la realización y la evaluación de producto al servicio de reciclaje (o continuidad del producto) (Bausela, 2003).

Por su parte, Flórez (2003) menciona tres modelos evaluativos cualitativos que permiten la participación activa de los involucrados en la evaluación, a través de opiniones, contextos, expectativas acerca del desempeño presente y futuro del currículo. Al igual que el modelo anterior CIPP, estos modelos no se basan en los resultados finales, sino en los procesos que ocurren en el desarrollo del currículo.

Asimismo, Flórez (2003) menciona que para hablar de estos tres modelos es necesario definir currículo, para precisar sobre lo que va a evaluarse. En términos generales, un currículo "es la manera de aplicar la teoría pedagógica en el aula de la enseñanza real... es la mediación entre la teoría y la realidad de la enseñanza, es el plan de acción que desarrolla el profesor con sus alumnos en el aula; es una pauta ordenadora del proceso de enseñanza" (Flórez, 2003, p.82). En otras palabras, es la acción que se lleva a cabo dentro de las aulas a partir de una teoría que pretende mejorar la realidad de lo que se enseña, no es solo teoría, es la acción que responde a la teoría, para mejorar la educación del alumnado.

En relación con lo anterior, señala además, que un plan de estudios "es apenas un esquema para distribuir contenidos, temas, materias y cursos según grados, niveles y prerrequisitos" a diferencia del currículo "que implica una concepción más amplia acerca de los contenidos, las experiencias y la actuación y secuencia para que los alumnos alcancen las metas de formación" (Flórez, 2003, p.82).

En ese mismo sentido, se debe tomar en cuenta el proceso de enseñanza que según Flórez (2003) es un encuentro real entre los sujetos que interactúan y se comunican a propósito de un plan, para generar aprendizajes. Indica "por ello es proceso y un producto a la vez, evaluable 
de manera inmediata y específica, o de manera global e integral (...) menciona además, que "el desarrollo es también producto y proceso a la vez" (p. 84) y por último, alude que:

El proceso del desarrollo y de formación individual y grupal de los alumnos alcanzado mediante la implementación del currículo genera un producto final que es el impacto social o repercusión de la ganancia del aprendizaje y formación sobre el desarrollo comunitario (Flórez, 2003, p. 84).

Dado que estos tres modelos se basan en la forma de evaluación de currículo, dentro del estudio, la integración de los tres modelos, forman el que es llamado modelo de evaluación curricular, pues pretende evaluar características que intervengan en la utilización y desarrollo del programa como parte del currículo.

Por último, está el modelo de evaluación de la gestión escolar donde la evaluación se concibe como el modelo, el dispositivo de principios que orientan la intención evaluadora y las indicaciones, en relación con los ámbitos de evaluación, con los momentos, las fuentes e instrumentos que se pueden utilizar, los procedimientos y los agentes que intervienen en ellos (Antúnez et al., 2009, p. 125).

Cada modelo antes señalado, se divide en diferentes categorías para el estudio, entre las cuales están: las características de los modelos, el ámbito de los modelos y la percepción que tienen los docentes y directora acerca del modelo de evaluación institucional.

\section{Metodología de la investigación}

El estudio responde a una investigación de tipo descriptivo. Para la recolección de datos se empleó un único cuestionario, el cual responde a los tres objetivos propuestos, el cual fue realizado por la investigadora y validado por investigadores de la Universidad de Costa Rica y por un grupo de docentes con características similares a las participantes en el estudio. El cuestionario fue entregado al personal docente y administrativo de cada institución seleccionada, se les dio un tiempo probable de entrega y se recogió cuando estaba listo.

El trabajo de investigación se desarrolló en varias fases, entre las cuales se presentan:

- Aprobación del anteproyecto de investigación.

- Carta de aprobación a la dirección de las instituciones designadas.

- Elaboración de los instrumentos de recolección de la información y su validación, para su posterior aplicación en los tres Jardines Independientes del Circuito 05 de la Dirección Regional de Educación de Cartago.

- Etapa de análisis de la información, contraponiendo la información de la gestión directiva 
con los docentes y la teoría.

- Posterior a los resultados obtenidos, se realizó una propuesta de modelo de evaluación de programas, como aporte al mejoramiento de la calidad de la educación en los centros infantiles de la región.

\section{Características de los modelos de evaluación en los Jardines de Niños en estudio}

En cuanto a las características de los móldelos, se permite evaluar los diferentes aspectos o elementos de un programa tales como contexto, objetivos, contenidos, situaciones de aprendizaje, actores; de los diferentes modelos de evaluación de programas elegidos para el estudio entre los cuales están el modelo CIPP, modelo curricular y modelo de gestión escolar.

En primer lugar, se analizaron las características del modelo CIPP en sus cuatro etapas, lo que permite evaluar el contexto para tomar decisiones sobre la designación de metas; la evaluación de entrada como ayuda para dar forma a las propuestas; la evaluación de proceso, como una guía para la realización, y la evaluación de producto al servicio de reciclaje (o continuidad del producto) (Bausela, 2003).

Posteriormente, se evaluó el modelo de evaluación curricular, Flórez (2003) los clasifica en tres modelos, Stake, Posner y Eisner, los cuales para efectos del estudio se utilizaron los tres en sus características generales como parte del modelo denominado evaluación curricular, estos son enfoques cualitativos que captan el movimiento, los procesos en acción, el caos y los sistemas borrosos, además, facilitan una percepción más amplia del proceso de identificar y decidir los objetivos, contenidos y estrategias de enseñanza que permita influir en los niños de manera positiva.

Este modelo a diferencia del CIPP no es por etapas, su principal valor lo posee la relación que tiene el currículo con los actores que participan en el proceso de aprendizaje como lo es el alumno, la familia, los docentes, la comunidad y demás personas cercanas; parte de un diagnóstico del contexto, una realidad del niño y de allí se planifican los aprendizajes no solo dentro del aula, sino en conjunto con los actores participantes de su realidad inmediata.

Por último, se revisó el modelo de la gestión escolar, el cual según lo indican Antúnez et al. (2009) pretende que "el centro lleve a cabo un proceso de autoevaluación una vez al año...en sus fases de sensibilización, planificación, diagnóstico, interpretación y propuesta de plan de mejora, de desarrollo durante las últimas semanas del año escolar" (p. 138).

Este modelo pretende rescatar la autoevaluación como una oportunidad para que el centro escolar mejore, para esto se necesita el trabajo compartido de los docentes en relación con el centro escolar, en cuanto al programa, el alumno y la institución por medio de un proceso 
de autoevaluación.

En este modelo se toma en cuenta el diseño del plan basado en el programa, la toma de decisiones en cuanto al currículo a nivel institucional, la correspondencia entre normas y valores institucionales, el buen uso de recursos materiales, el tiempo y el espacio, la planificación de metas de un grado a otro o de una institución a otra, las buenas relaciones entre personal docente, gestión escolar y familias, y una cultura evaluativa a nivel del centro escolar. Estas características son determinadas por diferentes áreas del modelo las cuales son el ámbito académico, ámbito administrativo, ámbito organizativo, ámbito de sistema de relaciones, ámbito de programas, proyectos y servicios complementarios.

En cuanto a los resultados obtenidos en las tres instituciones, sobre si estaban o no presentes las diferentes características de los modelos para responder al objetivo: Definir los modelos de la evaluación curricular aplicados por la gestión a los programas educativos, se evidenció lo siguiente:

Tabla 1: Características de los modelos presentes en los tres jardines de niños y niñas del Circuito 05 de la Dirección Regional de Cartago, 2012

\begin{tabular}{|c|c|c|c|}
\hline Institución & $\begin{array}{c}\text { Modelo de } \\
\text { Evaluación CIPP }\end{array}$ & $\begin{array}{l}\text { Modelo de } \\
\text { Evaluación } \\
\text { Curricular }\end{array}$ & $\begin{array}{l}\text { Modelo de Evaluación } \\
\text { de la Gestión Escolar }\end{array}$ \\
\hline $\begin{array}{c}\text { Jardín de Niños República } \\
\text { Francesa }\end{array}$ & $\begin{array}{l}\text { Parcialmente } \\
\text { presente }\end{array}$ & No está presente & Parcialmente presente \\
\hline $\begin{array}{l}\text { Jardín de Niños Juan } \\
\text { Vázquez de Coronado }\end{array}$ & $\begin{array}{l}\text { Parcialmente } \\
\text { presente }\end{array}$ & Parcialmente presente & Parcialmente presente \\
\hline $\begin{array}{c}\text { Jardín de Niños Carlos } \\
\text { Peralta }\end{array}$ & Totalmente presente & No está presente & No está presente \\
\hline
\end{tabular}

Nota: Carmona, 2012. Estudio realizado en tres jardines infantiles del Circuito 05, de la Dirección Regional de Educación de Cartago.

\section{Ámbitos del modelo de evaluación de programas}

Los ámbitos de los modelos de evaluación de programas que fueron considerados dentro del estudio, según Antúnez et al. (2009), se definen como "cada una de las áreas en que se puede agrupar las diferentes acciones y prácticas educativas que tienen lugar en el centro escolar" (p.130). Las áreas que se desarrollan en el estudio corresponden a las acciones y prácticas que tienen que ver con lo pedagógico que comprende en el centro escolar. 
Tabla 2: Ámbitos de los modelos de evaluación de programas de los modelos CIPP, curricular y de la gestión escolar aplicados en los jardines infantiles del Circuito 05 de la Dirección Regional de Educación de Cartago, 2012

\begin{tabular}{ccc}
\hline Modelo de Evaluación CIPP & $\begin{array}{c}\text { Modelo de Evaluación } \\
\text { Curricular }\end{array}$ & $\begin{array}{c}\text { Modelo de la Evaluación de } \\
\text { la Gestión escolar }\end{array}$ \\
\hline Contexto & Objetivos de aprendizaje & $\begin{array}{c}\text { Académico } \\
\text { Administrativo } \\
\text { Organizativo } \\
\text { Entrada }\end{array}$ \\
Proceso & Contenidos & $\begin{array}{c}\text { Sistema de relaciones } \\
\text { De los programas, proyectos y } \\
\text { servicios complementarios }\end{array}$ \\
\hline Producto & Estrategias de enseñanza & \\
\hline
\end{tabular}

Nota: Carmona, 2012. Estudio realizado en tres jardines infantiles del Circuito 05, de la Dirección Regional de Educación de Cartago

Cada modelo de evaluación de programas se comprende de varias características para identificar los ámbitos, las cuales se encuentran indicadas en la siguiente tabla:

Tabla 3. Características para la identificación de los ámbitos del modelo de evaluación de programas aplicados en los jardines infantiles del Circuito 05 de la Dirección Regional de Cartago, 2012

\begin{tabular}{lll}
\hline Modelo de evaluación CIPP & \multicolumn{1}{c}{$\begin{array}{c}\text { Modelo de evaluación } \\
\text { curricular }\end{array}$} & $\begin{array}{c}\text { Modelo de la evaluación de la } \\
\text { gestión escolar }\end{array}$ \\
\hline $\begin{array}{l}\text { Relación entre el diagnóstico y } \\
\text { el plan. }\end{array}$ & $\begin{array}{l}\text { Relación existente entre lo que } \\
\text { se enseña y el contexto. }\end{array}$ & $\begin{array}{l}\text { Relación entre lo que promueve la } \\
\text { institución y lo que dice el programa } \\
\text { preescolar. }\end{array}$ \\
$\begin{array}{l}\text { Evaluación de metas y } \\
\text { objetivos. }\end{array}$ & $\begin{array}{l}\text { Relación entre el currículo y las } \\
\text { necesidades del niño. }\end{array}$ & $\begin{array}{l}\text { Participación activa por parte del } \\
\text { personal de la institución. }\end{array}$ \\
$\begin{array}{l}\text { Evaluación de las limitaciones } \\
\text { de personal, recursos } \\
\text { materiales. }\end{array}$ & $\begin{array}{l}\text { Relación entre el currículo y los } \\
\text { valores de la institución. }\end{array}$ & $\begin{array}{l}\text { Aprovechamiento del espacio, } \\
\text { material y tiempo institucional en los } \\
\text { aprendizajes. }\end{array}$ \\
$\begin{array}{l}\text { Proceso de evaluación para } \\
\text { toma de decisiones. }\end{array}$ & $\begin{array}{l}\text { Relación entre el currículo y la } \\
\text { participación de la comunidad. }\end{array}$ & $\begin{array}{l}\text { Relación entre el personal docente, la } \\
\text { gestión escolar y las familias. }\end{array}$ \\
$\begin{array}{l}\text { Resultados del plan para } \\
\text { mejorar. }\end{array}$ & & Evaluación de los procesos. \\
\hline
\end{tabular}

Nota: Carmona, 2012. Estudio realizado en tres jardines infantiles del Circuito 05, de la Dirección Regional de Educación de Cartago. 
En esta parte de la investigación los docentes participantes deben identificar los ámbitos del modelo o los modelos de evaluación que se usa mayormente en la institución señalando por medio de las características que ellos mismos consideran se presentan en la institución.

En el primer modelo se tomaron en cuenta diferentes características que tratan de responder a los ámbitos del modelo de evaluación CIPP, entre las cuales se destaca la relación entre el diagnóstico y plan institucional; la evaluación de metas y objetivos a lo largo del ciclo lectivo; la evaluación de las limitaciones de personal y recursos materiales al principio del año y durante este; el proceso de evaluación para toma de decisiones con todo el personal de la institución y los resultados del plan para mejorar dentro de la institución.

El segundo modelo, de evaluación curricular, se divide en tres ámbitos los cuales son los objetivos del aprendizaje, es decir, la evaluación se centra en los logros y resultados reales de desempeño alcanzados a la luz de los objetivos en cada programa. Los contenidos que son los que fundamentan el currículo en cuanto a lo que se va a enseñar y por último las estrategias de enseñanza, las cuales son las experiencias que el estudiante tiene durante su jornada escolar, si son auténticas, interesantes, apropiadas para la edad, saludables, seguras, si abren nuevos horizontes cognitivos a los alumnos, si comprometen su actividad presente y futura (Flórez, 2003). Este modelo pretende mantenerse en una estrecha retroalimentación rediseñando el currículo según lo evaluado en función del cumplimiento de los objetivos, contenidos y estrategias.

En relación con el modelo de evaluación curricular para responder a estos tres ámbitos se tomaron en cuenta varias características que definen si se cumple cada uno de ellos. Sin embargo, los tres están íntimamente relacionados, pues se conciben como un proceso que se mantiene en constante evaluación para su cumplimiento. Entre estas características están: la relación existente entre lo que se enseña y el contexto; la relación entre el currículo y las necesidades del niño; la relación entre el currículo y los valores de la institución; la relación entre el currículo y la participación de la comunidad.

Estos ámbitos del modelo pretenden que se tome en cuenta el proceso que se deriva de los objetivos, contenidos y estrategias de enseñanza, que según Flórez (2003):

Es un encuentro real entre los sujetos que interactúan y se comunican a propósito de un plan, para generar aprendizajes. Por ello es proceso y un producto a la vez, evaluable de manera inmediata y específica, o de manera global e integral (...) El desarrollo es también producto y proceso a la vez. El proceso del desarrollo y de formación individual y grupal de los alumnos alcanzado mediante la implementación del currículo genera un producto final que es el impacto social o repercusión de la ganancia del aprendizaje y formación sobre el desarrollo comunitario. (p. 84) 
En el tercer modelo de evaluación de programas, se tomaron en cuenta diferentes ámbitos (académico, administrativo, organizativo, sistema de relaciones, y programas, proyectos y servicios complementarios), los cuales tratan de responder al modelo de evaluación de la gestión escolar por medio de las características que los definen, entre las cuales están la relación entre lo que promueve la institución y lo que dice el programa preescolar; la participación activa por parte del personal de la institución; el aprovechamiento del espacio, material y tiempo institucional en los aprendizajes; la relación entre el personal docente, la gestión escolar y las familias; y por último, la evaluación de los procesos.

En cuanto a los resultados obtenidos en las tres instituciones sobre si estaban o no presentes las características de los diferentes ámbitos de los modelos en estudio, para responder al objetivo: Identificar los ámbitos de evaluación de programas presentes en el modelo de evaluación curricular aplicado por la gestión educativa de los jardines independientes de la Dirección Regional de Cartago, se evidenció lo siguiente:

Tabla 4. Ámbitos de los modelos presentes en los tres jardines de niños y niñas del circuito 05 de la dirección Regional de Cartago, 2012

\begin{tabular}{|c|c|c|c|}
\hline Institución & $\begin{array}{c}\text { Modelo de } \\
\text { Evaluación CIPP }\end{array}$ & $\begin{array}{l}\text { Modelo de } \\
\text { Evaluación } \\
\text { Curricular }\end{array}$ & $\begin{array}{l}\text { Modelo de } \\
\text { Evaluación de la } \\
\text { Gestión Escolar }\end{array}$ \\
\hline $\begin{array}{c}\text { Jardín de Niños República } \\
\text { Francesa }\end{array}$ & Parcialmente presente & No está presente & No está presente \\
\hline $\begin{array}{l}\text { Jardín de Niños Juan Vázquez de } \\
\text { Coronado }\end{array}$ & Totalmente presente & $\begin{array}{l}\text { Parcialmente } \\
\text { presente }\end{array}$ & Parcialmente presente \\
\hline Jardín de Niños Carlos Peralta & Totalmente presente & No está presente & No está presente \\
\hline
\end{tabular}

Fuente: Carmona, 2012. Estudio realizado en tres jardines infantiles del Circuito 05, de la Dirección Regional de Educación de Cartago.

\section{Percepción que tiene el personal docente en relación con el modelo aplicado por la gestión en cada centro}

Esta categoría del estudio pretende mostrar cuál es la valoración que tiene el personal acerca del modelo de evaluación de programas curriculares que se lleva a cabo en el centro para el cual labora. 
Dentro de esta investigación, se entiende la percepción como la valoración que tienen las docentes de la gestión que se realiza en el centro educativo en cuanto a las siguientes afirmaciones, que son parte de un esperado modelo de evaluación en cualquier centro educativo, pues busca la mejora de este en relación con el alumnado, los aprendizajes, el programa, los logros, las limitaciones, las metas, los objetivos, la familia y la comunidad.

- Diseño del plan que responde a las necesidades del niño desde el programa de preescolar.

- Proceso de evaluación del plan para mejorar los aprendizajes.

- Participación docente en la toma de decisiones que favorezcan la mejor aplicación del programa preescolar.

- Participación de la familia y comunidad.

- Resultados de logros.

- Limitaciones.

- Metas y objetivos.

En relación con lo anterior, la gestión de los centros ha de estar íntimamente ligada entre lo administrativo y lo pedagógico, buscando convertir la institución en un lugar que promueva el cambio que la sociedad requiere, en la búsqueda de una mejor educación para todos, sustentada en el uso activo de la investigación educativa de la evaluación y rendición de cuentas. Con respecto a esto, el Consejo Superior de Educación (2008) señala:

Los centros educativos de calidad se identificarán, precisamente, mediante los resultados de los procesos de evaluación integral de la educación: la evaluación institucional, la evaluación del currículo nacional básico, de los planes y currículos enriquecidos dispuesto por cada centro educativo, del desempeño del recurso humano, de los aprendizajes de los estudiantes y del impacto de la educación en el desarrollo del país y la calidad de la convivencia. (p. 24)

De acuerdo con lo anterior, se puede decir que la gestión del centro tiene un papel muy importante en relación con la evaluación de programas, como parte de las funciones del ámbito de evaluación que le corresponde aplicar todo esto con el fin de mejorar la calidad de la educación en beneficio de los niños y niñas del centro que gestiona y con ello contribuye al desarrollo nacional.

Se observa en estas instituciones, la percepción que las docentes tienen de acuerdo con la labor de la gestión dentro del centro escolar, en relación con la puesta en práctica del programa de preescolar y la evaluación que se lleva a cabo dentro del centro educativo sobre el mismo, con la finalidad de mejorar la educación dentro de la institución y a la vez, como parte de un gran 
sistema educativo que pretende mejorar la educación, en el nivel nacional.

Según la percepción de las docentes y administradoras de los diferentes centros educativos, considerando los aspectos dentro de las organizaciones educativas que indican que se puede evaluar todos los procesos que en ella se ejecuten, ya sean procesos de enseñanza aprendizaje, proyectos, personal docente, recursos, programas, entre otros; por parte de la gestión que se realiza en el centro educativo todo esto como parte de una verdadera evaluación.

En cuanto a los resultados obtenidos en las tres instituciones sobre la percepción de las docentes y administradoras en relación con la gestión del centro y el modelo de evaluación utilizado, para responder al objetivo: Describir la percepción que tienen los docentes sobre el modelo de evaluación curricular que aplica la gestión educativa de la institución de los jardines independientes del circuito 05 de la Dirección Regional de Educación de Cartago, se evidenció lo siguiente:

Tabla 5. Percepción que tiene el personal docente en relación con el modelo aplicado por la gestión en cada centro de los jardines infantiles del Circuito 05 de la Dirección Regional de Educación de Cartago, 2012

\begin{tabular}{cl}
\hline Institución & \multicolumn{1}{c}{ Percepción } \\
\hline Jardín de Niños República Francesa & $\begin{array}{l}\text { No hay claridad en la percepción que tienen las docentes en cuanto } \\
\text { al modelo de evaluación aplicado por la gestión. }\end{array}$ \\
Jardín de Niños Juan Vázquez de & $\begin{array}{c}\text { No hay claridad en la percepción que tienen las docentes en cuanto } \\
\text { Coronado }\end{array}$ \\
Jardín de Niños Carlos Peralta & $\begin{array}{l}\text { Se considera que el modelo de evaluación aplicado por la gestión } \\
\text { promueve la mejora constante en el programa, la institución, la } \\
\text { familia, la comunidad. }\end{array}$ \\
\hline
\end{tabular}

Fuente: Carmona, 2012. Estudio realizado en tres jardines infantiles del Circuito 05, de la Dirección Regional de Educación de Cartago.

Se observa que de las tres instituciones, solo en el Jardín de Niños Carlos Peralta, el personal tiene una clara percepción acerca del tipo de modelo de evaluación que se aplica en la institución. El Jardín de Niños República Francesa no manifiesta congruencia entre todas las participantes acerca del modelo de evaluación que la institución tiene y en el Jardín de Niños Juan Vázquez de Coronado, solo una docente considera que el modelo aplicado no siempre refleja las características deseables.

La gestión educativa es la encargada de llevar a cabo el cumplimiento de la política educativa, y para hacerla posible necesita de una evaluación constante e integral, que permita recabar la información necesaria para la toma de decisiones acertadas. En relación con esto, el 
Consejo Superior de Educación (2008) menciona:

La exitosa ejecución de una política educativa encuentra su apoyo e impulso en el andamiaje de información, administración e instrumentos necesarios para la operacionalización y la adecuación de los lineamientos y acciones de que dispone esa política. Es igualmente indispensable la evaluación integral permanente y, consecuentemente, la introducción de las acciones correctivas que permitan el logro de objetivos esenciales programados. (p. 21)

Según se cita anteriormente, la evaluación como herramienta de la gestión permite que se cumpla con éxito los objetivos de la política educativa. De esta manera, la gestión juega un papel muy importante en todo centro educativo, integrando los aprendizajes, con el desarrollo personal y colectivo de los estudiantes. Al respecto, el Consejo Superior de Educación (2008) indica:

La única forma de lograr que la política educativa alcance efectivamente los objetivos que propone, es mediante una gestión que logre que los procesos y las acciones de todo el sistema educativo se mantengan orientados siempre a los fines que se buscan: el aprendizaje y el desarrollo personal y colectivo de los y las estudiantes promovido y facilitado por la calidad de los centros educativos. (p. 22)

La gestión del centro es pues, la encargada de que el sistema educativo logre mejoras constantes en la educación, buscando la calidad, desde el centro, poniendo en práctica los programas que el MEP ofrece, pero que estos a la vez, se contrapongan con la realidad del niño de manera que lo que se haga traiga beneficios a todos los estudiantes en su contexto real.

En relación con esto, se puede decir que el programa no puede tener sus logros por sí solo, sino que necesita hacerlo real al contexto del niño y según se observa en el estudio, solo cuando esta relación es aplicada tiene los resultados esperados, como lo demuestra el Jardín de Niños Carlos Peralta.

La oportunidad que brinda un modelo de evaluación de programas, ya sea los propuestos en este estudio, o en algún otro, debe demostrar que la institución siempre está en continua mejora, según el estudio al no haber un determinado modelo de evaluación de programas, no hay congruencia entre las participantes de lo que se está haciendo o no, dentro de la institución y hacia dónde va esta.

La gestión del centro es la responsable de poner en práctica el modelo de evaluación de programas que busque la mejora institucional dentro de un gran sistema nacional. Se observa que el programa al hacerlo parte del contexto real del niño, logra las mejoras institucionales que se desean. 
El programa de preescolar se rige bajo los principios del desarrollo humano infantil que sustentan la educación preescolar, se derivan de investigaciones realizadas por estudiosos del desarrollo y el aprendizaje infantil, su puesta en práctica debe considerar tanto las características de la niñez, la familia, el equipo docente y administrativo, como del contexto sociocultural en que se desenvuelve la acción educativa, es por esto que la gestión debe tomar en cuenta dentro de su trabajo el programa como base en conjunto con la realidad del niño, para que se logre lo que realmente se espera en su desarrollo y contexto sociocultural.

El programa de preescolar responde a los fines de la educación preescolar que se contempla en la Ley Fundamental de la Educación (1957) citada por Delgado (1999), es por esto que ninguna institución puede dejar de lado el programa y dedicarse a solo hacer lo que se vaya presentando; es decir, solucionando problemas sin preverlos primero; los objetivos de la educación preescolar pretenden:

- Propiciar el desarrollo integral de los niños atendiendo las áreas cognoscitivo-lingüístico, socioemocional y psicomotriz, para una mejor calidad de vida como ser individual y social.

- Favorecer el desarrollo socioemocional del niño, mediante la formación de hábitos para la convivencia social, así como valores y actitudes que le permitan interactuar positivamente con su medio cultural.

- Promover en el niño el desarrollo de destrezas y habilidades básicas, para el desarrollo óptimo de sus potencialidades.

- Estimular el desarrollo de la capacidad creadora para enriquecer la libre expresión de la personalidad infantil.

- Favorecer el desarrollo de actitudes científicas para asumir una posición crítica ante la vida.

- Promover en los actores sociales una actitud de afecto, respeto y protección para preservar y conservar su ambiente natural, social y cultural.

- Generar conciencia en los padres y la familia, de su papel en el desarrollo integral de los hijos, para su realización como personas y ciudadanos capaces de asumir la vida responsablemente en una sociedad democrática.

- Educar para la convivencia social, según los derechos y libertades fundamentales enunciados en la Declaración de los Derechos del Niño. (pp. 3-4)

La gestión de la institución no puede olvidar que se parte de un sistema integral en el nivel nacional en la educación; salirse de este sistema provoca que no haya concordancia con lo que se enseña y se espera que se promueva como parte de un ciudadano costarricense.

Los logros que las instituciones vayan teniendo se debe a una buena gestión por parte de la administradora; una herramienta para esto es la evaluación del programa durante el año 
lectivo, donde se contemple características como las de los modelos anteriormente estudiados, en el cual se promueva una acción educativa basada en el programa y en la realidad del niño.

La gestión del centro se vuelve un reto para cada administrador, principalmente en instituciones con matrícula muy amplia, pero con un buen equipo de trabajo por parte del personal, esto se logra, como se observó en el Jardín de Niños Carlos Peralta, en el cual la relación con las docentes siempre se presenta y las participantes son tomadas en cuenta siempre, como parte de la toma de decisiones a nivel institucional.

Esta buena gestión, se logra a través de la evaluación constante dentro del centro, para así observar lo que está fallando dentro de los procesos de aprendizaje y el uso de los recursos humanos, materiales, pedagógicos, culturales y demás. Todo está íntimamente relacionado, la gestión del centro en conjunto con la evaluación tiene como función detectar estas limitaciones para que en integración con el personal docente, familia y comunidad se conviertan en soluciones y logros para una mejor educación.

La gestión del centro tiene un papel muy importante en la evaluación de programas, si esta se lleva a cabo con seriedad y continuidad, los resultados serán siempre los mejores para todos, en especial para los niños y niñas costarricenses.

Como parte del estudio, se llega a las siguientes conclusiones, en relación con la presencia de los modelos de evaluación de programas presentes en cada institución.

\section{Conclusiones}

En el Jardín de Niños República Francesa, los modelos de evaluación identificados son el modelo de evaluación CIPP y el modelo de evaluación de la gestión escolar. No obstante, no hay congruencia sobre la percepción del manejo de un modelo de evaluación específico que aplique la gestión de la institución en la evaluación de programas, para mejorar la educación que se ofrece a la población estudiantil que se atiende en el centro.

El Jardín de Niños Carlos Peralta identificó el modelo de evaluación CIPP, las participantes concuerdan en que el modelo de evaluación aplicado por la gestión siempre busca las mejoras. Se evidencia una clara relación desde el principio del estudio en que las características y ámbitos del modelo CIPP están presentes como parte de la gestión del centro.

El Jardín Juan Vásquez de Coronado considera que ámbitos y características de todos los modelos se encuentran presentes en la institución pero no en su totalidad, por lo que no se identifica un modelo de evaluación de programas en específico. Una de las tres docentes no está de acuerdo en que haya un modelo de evaluación que busque las mejoras aplicado por la gestión, por lo que debe elegirse un modelo y fortalecerlo como parte de la evaluación de programas que la gestión del centro debe aplicar. 
Dentro del estudio, el Jardín de Niños Carlos Peralta, fue la única institución donde las participantes identificaron un modelo de evaluación aplicado por la institución el cual corresponde al modelo CIPP y la percepción que tienen acerca de este modelo es congruente, pues refleja por medio de todas las participantes que se aplica de forma constante de manera que se logre mejoras para la institución siempre. Este Jardín de Niños logró identificar por parte de todas las participantes, tanto las características como los ámbitos, del modelo de evaluación de programas aplicado por la gestión de la institución. Además, se observa que hubo congruencia en esta institución entre la identificación del modelo y la forma en que las docentes perciben la aplicación de un modelo de evaluación de programas por parte de la gestión del centro.

El Jardín de Niños Carlos Peralta es la institución que maneja un modelo de evaluación de programas, el cual responde al modelo de evaluación CIPP; además, tiene en su ambiente escolar según la percepción de las participantes, un modelo que busca una relación de mejora entre lo que dice el programa de preescolar y la realidad del niño de manera continua, dado que siempre se presentan los criterios del modelo de evaluación empleados por la gestión centro.

En cuanto al Jardín República Francesa, el estudio demuestra que el modelo de evaluación aplicado por la gestión busca mejoras institucionales entre el programa de preescolar y la realidad del niño. Se muestra una necesidad por fortalecer las características y ámbitos de los modelos de evaluación de programas por parte de la gestión del centro y adoptar un modelo de evaluación de programas que permita brindar un servicio de calidad para la población infantil del centro.

El Jardín Juan Vázquez de Coronado, determina que no existe un modelo definido por la gestión para evaluar los programas, pues el estudio comprueba que hay presencia de las características y de los ámbitos de todos los modelos de evaluación de programas de forma parcial o nula, esto se refleja también, en la percepción que tienen las docentes sobre el modelo aplicado, en el cual tampoco hay concordancia entre las participantes como parte de la existencia de un modelo de evaluación de programas que busque la mejora del centro, por lo tanto se debe adoptar un modelo de evaluación de programas que sirva como herramienta para mejorar el servicio educativo que se brinda en la institución.

La gestión del centro en su buena práctica debe reforzar su estructura de evaluación por medio de un modelo de evaluación de programas determinado, que promueva la mejora del centro por medio de la aplicación del programa del ciclo de transición en relación con la institución, la familia, la comunidad, atendiendo las necesidades del niño estipuladas desde el inicio del año en un plan institucional.

La calidad de la educación solo tendrá sus mejoras, en la medida en que docentes y administrativos se mantengan evaluando lo que pasa en sus centros educativos, se hace necesario el uso de la evaluación de programas; sin embargo, esto es responsabilidad de todos: 
institución, familia y comunidad, todos trabajando en objetivos y metas comunes, lograrán una mejor educación y por ende un mejor bienestar del niño y la sociedad costarricense.

\section{Referencias}

Antúnez, S., Carnicero, P. y Silva, P. (2009) Modelo Regional de Gestión Escolar en Centroamérica y República Dominicana: análisis de la situación y propuesta para la convergencia regional. España: Universidad de Barcelona.

Aragón, F. (2005). La administración educativa: implicaciones y críticas en torno a su definición. Revista electrónica Visión Educativa. Secretaria de Educación y Cultura del Estado de Sonora (SEC), México. Recuperado de: www.sec-sonora.gob.mx./ve/2.html

Arroyo V., J.A. (2009). Administración de la educación. Recuperado de: http://www.slideshare.net/ Jarval/administracion-de-la-educacin-1671803

Bausela, E. (2003). Metodología de la investigación evaluativa: Modelo CIPP. Revista Complutense de Educación, 14(2), pp. 361-376. Recuperado de: http://revistas.ucm.es/edu/11302496/ articulos/RCED0303220361A.pdf

Consejo Superior de Educación [CSE]. (2008). El centro educativo de calidad como eje de la educación costarricense. San José, Costa Rica: MEP.

Correa, C. (2004). Gestión y evaluación de la calidad en la educación: referentes generales para la acreditación. Bogotá, Colombia: Cooperativa Editorial Magisterio.

Delgado, E. (1999). Planeamiento didáctico en la educación preescolar. San José, Costa Rica: EUNED.

Flórez, R. (2003). Docente del siglo XXI. Evaluación pedagógica y cognición. Colombia: Mc Graw-Hill Interamericana.

Ministerio de Educación Pública [MEP]. (1996). Programa de estudio Ciclo de Transición. San José, Costa Rica: Autor.

Nieto, J. M. (2003). La evaluación del centro escolar como proceso de mejora. En: González, M.T. (Coord.) Organización y gestión de centros escolares: dimensiones y procesos (pp. 265-287). España: Pearson Educación.

Pérez, J. (2000). La evaluación de programas educativos: conceptos básicos, planteamientos generales y problemática. Revista de Investigación Educativa, 18(2), pp. 261-287. Recuperado de: http://www.uned-terrassa.es/docs biblioteca/perez juste.pdf 
Romero E., F. (2001). Supervisión y evaluación de centros educativos. San José, Costa Rica: MEP.

Ruíz, J. M. (1998). Cómo hacer una evaluación de centros educativos. Recuperado de: http:// books.google.es/books?hl=es\&lr=\&id=6yTipw09DVkC\&oi=fnd\&pg=PA11\&dq=evaluaci on+curricular+de+programas\&ots=oFzZJdMCLh\&sig=vluTM2lkOuOKUn5ehLSLmpttn$\underline{8 \# \mathrm{v}=\text { onepage } \& q \mathrm{f}=\text { false }}$ 